\title{
Incidentally detected pituitary macroadenoma in a case of neurofibromatosis type 1: A case report and review of literature
}

\author{
Himanshu Mishra', Rohit Kumar', Amit Kumar', Sanjay Kumar Suman ${ }^{3}$ \\ From ' ${ }^{1}$ unior Resident, ${ }^{2}$ Associate Professor, ${ }^{3}$ Professor and Head, Department of Radiology, Indira Gandhi Institute of Medical Sciences, Patna, \\ Bihar, India
}

\begin{abstract}
Neurofibromatosis is a cancer predisposition syndrome that leads to an increased risk of certain neural tumors. Neurofibromatosis type 1 (NF-1) presents with multiple neurofibromas which may be dermal or plexiform. Neurofibromatosis type 2 is characterized by bilateral vestibular schwannomas. Pituitary masses have been infrequently described in cases of NF-1. We describe the case of an incidentally discovered pituitary macroadenoma in a patient with NF-1. Apart from the macroadenoma, the patient had plexiform neurofibromas of the orbit, Lisch nodules of the iris, and café-au-lait spots. The patient was called for medical management but failed to show due to the COVID-19 pandemic.
\end{abstract}

Key words: Café-au-lait spots, Neurofibromatosis, Pituitary macroadenomas, Plexiform neurofibromas

$\mathrm{N}$ eurofibromatosis is a group of disorders affecting the growth and development of neural tissues. While neurofibromatosis type 1 (NF-1) is characterized by the presence of peripheral neurofibromas, the dominant feature of neurofibromatosis type 2 is schwannoma (especially vestibular schwannoma). NF-1 has an autosomal dominant mode of inheritance and is caused by the mutation of the NF-1 gene on chromosome 17 which encodes for a tumor suppressor gene, neurofibromin. It is a relatively common disease, with an estimated incidence of one in 3000 people. No sex predilection has been found [1-3].

The clinical manifestations include dermal and plexiform neurofibromas, cutaneous lesions (such as café-au-lait spots and axillary/groin freckling), ocular lesions (iris hamartomas and optic nerve gliomas), and bony dysplasias (such as sphenoid wing dysplasia and long bone cortical thinning). Numerous other lesions have been described in cases of NF-1 [1,4]. Pituitary macroadenomas are not usually seen in association with neurofibromatosis and are rarely reported in the literature. We describe such a case in our report.

A 17-year-old young female presented to the department of ophthalmology with a complaint of swellings around both eyes for 2 years. Initially, the swellings were small but gradually, increased in size. Her mother was a known case of NF-1.

\section{Access this article online}

Received - 11 May 2021

Initial Review - 26 May 2021

Accepted - 05 June 2021

DOI: 10.32677/IJCR.2021.v07.i06.008
On examination, the vitals were stable. Firm, non-tender, noncompressible, and non-fluctuant subcutaneous swellings were noted along the lateral canthus of the right as well as left eyes. Multiple cutaneous café-au-lait spots were also noted (Fig. 1). Ophthalmic examination revealed the presence of pigmented iris hamartomas.

Contrast-enhanced magnetic resonance imaging (MRI) of the brain and orbits was performed which revealed a poorly marginated avidly enhancing soft-tissue mass in the subcutaneous plane along the superolateral aspect of the right orbit. The soft-tissue mass was seen to extend into the right orbit and encase the superior and lateral rectus muscles. Another similar but smaller lesion was present along the lateral aspect of the left orbit. Clinical and radiological features were suggestive of plexiform neurofibromas (Figs. 2 and 3). An incidental finding was the presence of an avidly enhancing sellar mass measuring approximately $12 \mathrm{~mm}$
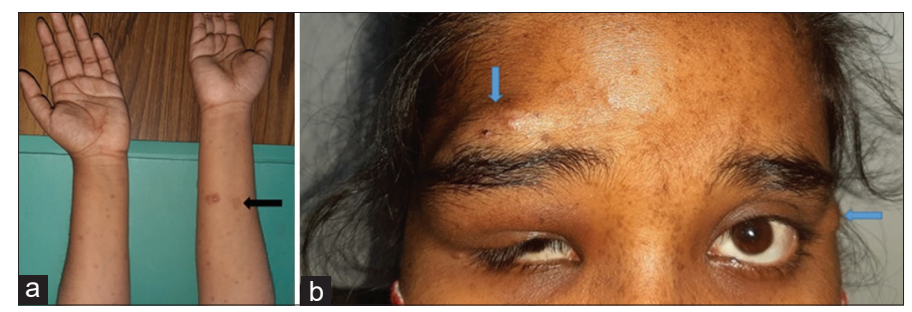

Figure 1: Clinical photographs showing multiple pigmented macules, also known as café-au-lait spots, on the palmar aspects of both forearms (one of the macules is indicated by the black arrow) (1a) and swellings in both the periorbital regions (1b)

Correspondence to: Dr. Himanshu Mishra, Department of Radiology, Indira Gandhi Institute of Medical Sciences, Patna, Bihar, India. E-mail: himanshu2505@ rediffmail.com

(C) 2021 Creative Commons Attribution-NonCommercial 4.0 International License (CC BY-NC-ND 4.0). 
$\times 12 \mathrm{~mm}$, which was not separable from the pituitary gland. Imaging features were suggestive of a pituitary macroadenoma (Figs. 4 and 5). Elevated serum prolactin $(238 \mathrm{mcg} / \mathrm{L})$ confirmed the diagnosis. The patient was called for medical management but failed to show due to the COVID-19 pandemic.

\section{DISCUSSION}

NF-1, also known as von Recklinghausen disease, is a phakomatosis affecting multiple organ systems. The genetic basis of the disease is the inactivation of the tumor suppressor gene neurofibromin on chromosome 17 leading to the overexpression of a potent oncogene Ras. This results in an increased risk of the development of neural tumors such as benign neurofibromas as well as malignant peripheral nerve sheath tumors [2].

Cutaneous lesions are ubiquitous manifestations of NF-1 and include café-au-lait spots (hyperpigmented light to dark brown macules) as well as axillary and groin freckling. Ophthalmic manifestations include pigmented iris hamartomas (Lisch nodules) and optic nerve gliomas. Most of the patients with NF-1 who had a coexistent pituitary mass presented with symptoms related to pituitary mass such as vision loss (due to mass effect),
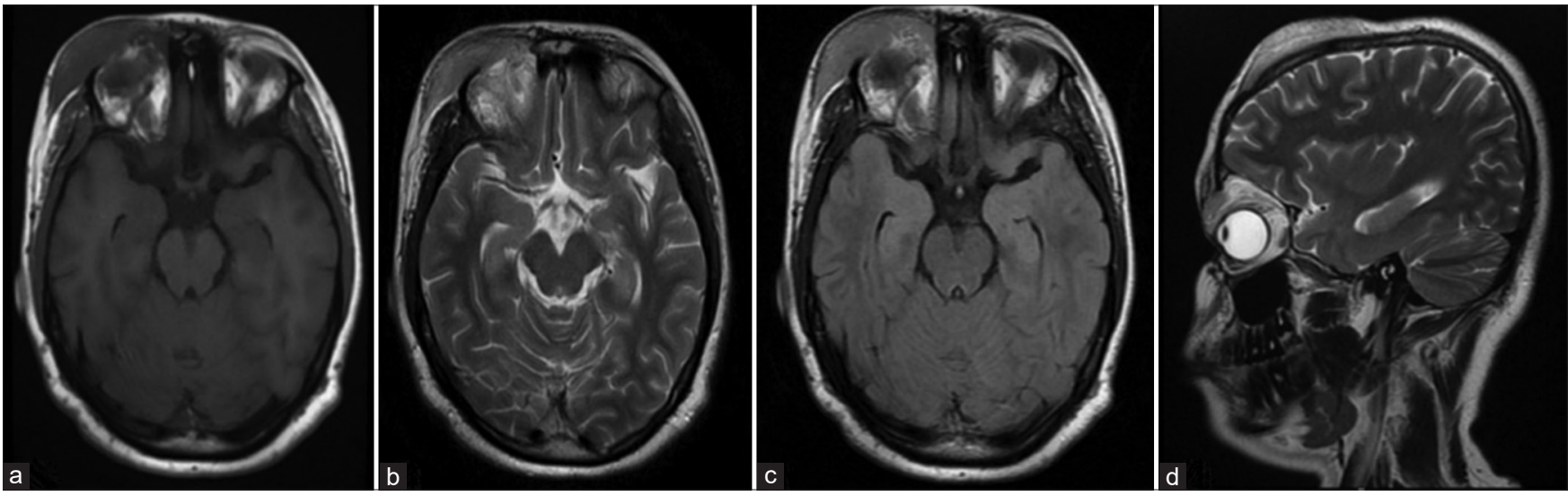

Figure 2: Non-contrast magnetic resonance images show a poorly marginated mass in the lateral aspect of right orbit which appears iso-tohypointense on T1 (2a), mildly hyperintense on T2 and FLAIR (2b and c). Sagittal T2 image shows another similar lesion in the scalp (2d). These are plexiform neurofibromas
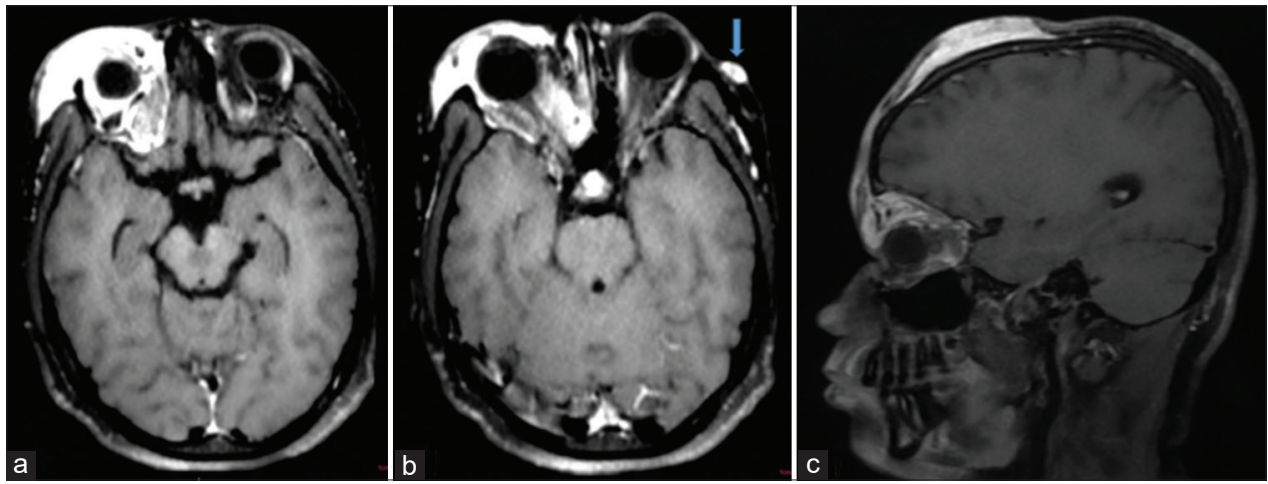

Figure 3: (a-c) Contrast-enhanced T1-FS images show that the neurofibromas are intensely enhancing. The right-sided lesion extends into the orbit. A smaller lesion is visible on the lateral aspect of the left orbit (blue arrow in $3 b$ )
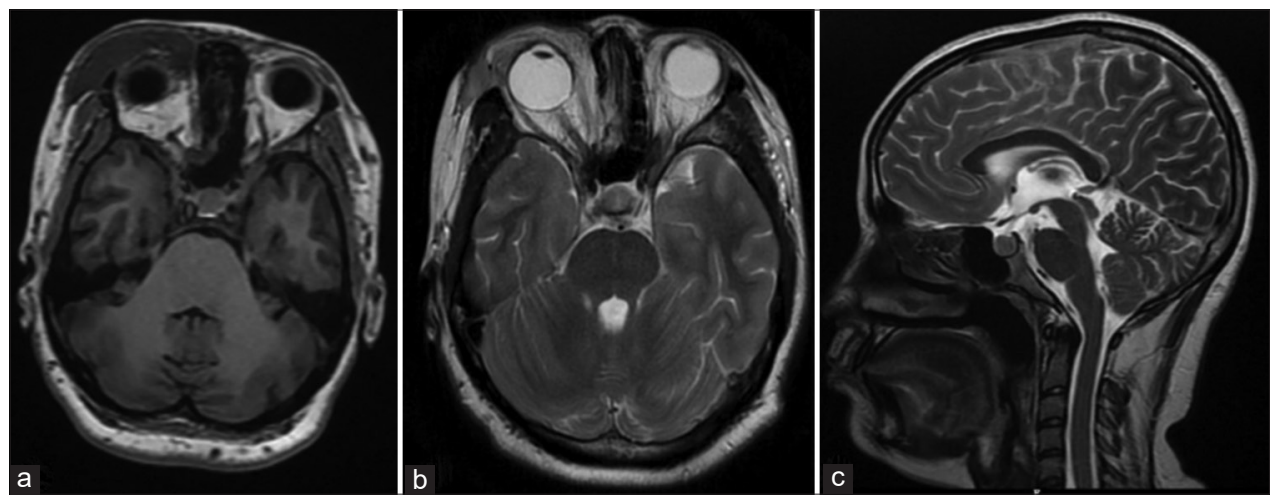

Figure 4: (a-c) A series of non-contrast magnetic resonance images show a sellar mass indistinguishable from the pituitary gland. This is a pituitary macroadenoma 


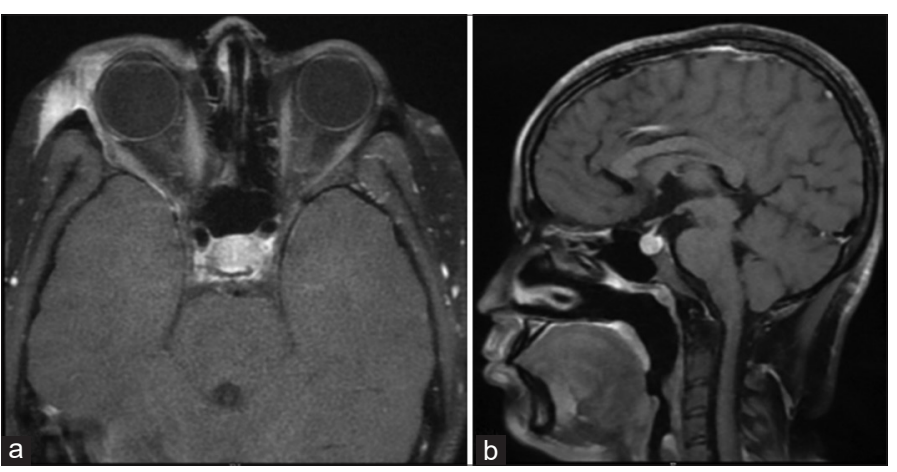

Figure 5: (a and b) A series of contrast-enhanced T1-FS images show that the macroadenoma is avidly enhancing

galactorrhea (due to prolactin excess), and acromegaly (in case of growth hormone-secreting tumors). In our patient, pituitary macroadenoma was an incidental finding and the patient was asymptomatic.

Numerous other manifestations have been described in NF-1 which include - but are not limited to - progressive stenosis of supraclinoid internal carotid arteries, dural ectasias, sphenoid wing dysplasia, cortical thinning of long bones, and pseudoarthrosis of tibias [4].

Neural tumors that have been described in NF-1 include dermal and plexiform neurofibromas. Dermal neurofibromas arise from the peripheral nerves and present as well-defined swellings over the body. On MRI, they appear as solitary or multifocal discrete round or ovoid scalp lesions with low signal on T1WI, high signal on T2WI, and intense enhancement on postcontrast images. Plexiform neurofibromas are usually seen in the orbit as poorly marginated serpentine masses infiltrating the orbit, extraocular muscles, eyelids, and the adjacent structures. On MRI, they appear to be of heterogeneous signal intensity and variable contrast enhancement [3].

Pituitary macroadenomas have been rarely described in the literature in association with NF-1. Hannah-Shmouni et al. [5], Garrido and del Pozo Picó [6], Nakajima et al. [7], and Pinnamaneni et al. [8] have described pituitary masses in patients with NF-1 in their respective case reports. As per the information available to the authors of this paper, no large-scale studies have been conducted to study the association between pituitary tumors and NF-1. Hence, it is not clear whether there is a definite association between pituitary tumors and NF-1. Pei and Melmed concluded that the inactivation of neurofibromin may not have a primary role in the formation of pituitary adenomas [9]. However, Caimari and Korbonits included NF-1 as a predisposing factor for pituitary adenomas [10].

\section{CONCLUSION}

As of now, there is insufficient evidence for a causal relationship between NF-1 and pituitary neoplasms. Nevertheless, anecdotal data suggest that there is some correlation between the two. Moreover, patients may be largely free of the symptoms of pituitary dysfunction, as our case demonstrates. Clinicians as well as radiologists ought to maintain a high index of suspicion to discover any hitherto undisclosed pituitary mass lesion in patients with NF-1.

\section{REFERENCES}

1. Gerber PA, Antal AS, Neumann NJ, Homey B, Matuschek C, Peiper M, et al. Neurofibromatosis. Eur J Med Res 2009;14:102-5.

2. Jett K, Friedman JM. Clinical and genetic aspects of neurofibromatosis 1. Genet Med 2010;12:1-11.

3. Friedman JM. Epidemiology of neurofibromatosis Type 1. Am J Med Genet 1999;89:1-6.

4. Boyd KP, Korf BR, Theos A. Neurofibromatosis Type 1. J Am Acad Dermatol 2009;61:1-14.

5. Hannah-Shmouni F, Demidowich AP, Rowell J, Lodish M, Stratakis CA. Large pituitary gland with an expanding lesion in the context of neurofibromatosis 1. BMJ Case Rep 2017;2017:222411.

6. Garrido CA, del Pozo Picó C. Acromegalia y neurofibromatosis Tipo 1. Es casual la asociación entre ambas entidades? Endocrinol Nutr 2013;60:144-5.

7. Nakajima M, Nakasu Y, Nakasu S, Matsuda M, Handa J. Pituitary adenoma associated with neurofibromatosis: Case report. Nihon Geka Hokan 1990;59:278-82.

8. Pinnamaneni K, Birge SJ, Avioli LV. Prolactin-secreting pituitary tumor associated with von Recklinghausen's disease. Arch Intern Med 1980;140:397-9.

9. Pei L, Melmed S. The neurofibromatosis gene in human pituitary adenomas. Endocr Pathol 1994;5:229-32.

10. Caimari F, Korbonits M. Novel genetic causes of pituitary adenomas. Clin Cancer Res 2016;22:5030-42.

Funding: None; Conflicts of Interest: None Stated.

How to cite this article: Mishra H, Kumar R, Kumar A, Suman SK. Incidentally detected pituitary macroadenoma in a case of neurofibromatosis type 1: A case report and review of literature. Indian J Child Reports. 2021;7(6):244-246. 